\title{
(อ) OPEN ACCESS \\ EULAR points to consider on pathophysiology and use of immunomodulatory therapies in COVID-19
}

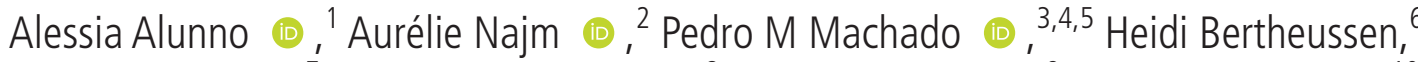 \\ Gerd R Burmester, ${ }^{7}$ Francesco Carubbi ${ }^{10},{ }^{8}$ Gabriele De Marco, ${ }^{9}$ Roberto Giacomelli, ${ }^{10}$

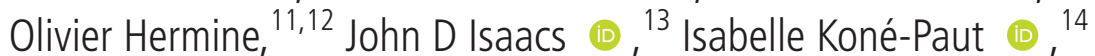 \\ César Magro-Checa, ${ }^{15}$ lain McInnes, ${ }^{2}$ Pier Luigi Meroni 이, ${ }^{16}$ Luca Quartuccio (ㄷ), ${ }^{17}$ \\ Athimalaipet V Ramanan, ${ }^{18,19}$ Manuel Ramos-Casals, ${ }^{20}$ Javier Rodríguez Carrio, ${ }^{21}$ \\ Hendrik Schulze-Koops (i), ${ }^{22}$ Tanja A Stamm (10, , ${ }^{23}$ Sander W Tas, ${ }^{24}$ \\ Benjamin Terrier (10 , ${ }^{25}$ Dennis G McGonagle, ${ }^{9}$ Xavier Mariette ${ }^{26}$
}

Handling editor Désirée van der Heijde

For numbered affiliations see end of article.

\section{Correspondence to}

Professor Xavier Mariette, Assistance Publique-Hôpitaux de Paris, Hôpital Bicêtre, INSERM UMR1184, Department of Rheumatology, Université Paris-Saclay, 91190 Saint-Aubin, Île-de-France, France: xavier.mariette@aphp.fr

$\mathrm{AA}$ and $\mathrm{AN}$ contributed equally.

Received 15 December 2020 Revised 11 January 2021 Accepted 27 January 2021 Published Online First 5 February 2021

\section{Linked}

- http://dx.doi.org/10.1136/ annrheumdis-2020-219725

- http://dx.doi.org/10.1136/ annrheumdis-2021-219957

Check for updates

(C) Author(s) (or their employer(s)) 2021. Re-use permitted under CC BY-NC. No commercial re-use. See rights and permissions. Published by BMJ.

To cite: Alunno A, Najm A, Machado PM et al. Ann Rheum Dis 2021:80:698-706.

\section{ABSTRACT}

Objectives Severe systemic inflammation associated with some stages of COVID-19 and in fatal cases led therapeutic agents developed or used frequently in Rheumatology being at the vanguard of experimental therapeutics strategies. The aim of this project was to elaborate EULAR Points to consider (PtCs) on COVID-19 pathophysiology and immunomodulatory therapies.

Methods PtCs were developed in accordance with EULAR standard operating procedures for endorsed recommendations, led by an international multidisciplinary Task Force, including rheumatologists, translational immunologists, haematologists, paediatricians, patients and health professionals, based on a systemic literature review up to 15 December 2020. Overarching principles (OPs) and PtCs were formulated and consolidated by formal voting.

Results Two OPs and fourteen PtCs were developed. OPs highlight the heterogeneous clinical spectrum of SARS-CoV-2 infection and the need of a multifaceted approach to target the different pathophysiological mechanisms. PtCs 1-6 encompass the pathophysiology of SARS-CoV-2 including immune response, endothelial dysfunction and biomarkers. PtCs 7-14 focus on the management of SARS-CoV-2 infection with immunomodulators. There was evidence supporting the use of glucocorticoids, especially dexamethasone, in COVID-19 cases requiring oxygen therapy. No other immunomodulator demonstrated efficacy on mortality to date, with however inconsistent results for tocilizumab. Immunomodulatory therapy was not associated with higher infection rates.

Conclusions Multifactorial pathophysiological mechanisms, including immune abnormalities, play a key role in COVID-19. The efficacy of glucocorticoids in cases requiring oxygen therapy suggests that immunomodulatory treatment might be effective in COVID-19 subsets. Involvement of rheumatologists, as systemic inflammatory diseases experts, should continue in ongoing clinical trials delineating optimal immunomodulatory therapy utilisation in COVID-19.

\section{INTRODUCTION}

The rapid worldwide spread of the SARS-CoV-2 has led to an unpreceded pandemic. SARS-CoV-2 infection leads to heterogeneous disease phenotypes,
Key messages

What is already known about this subject?

- SARS-CoV-2 infection is potentially life threatening with severe disease being associated with immune system activation with severe pneumonia and pulmonary immunothrombosis that may be amenable to immunomodulatory treatments which have largely been pioneered in the rheumatology therapeutic arena.

- The enormous amount of studies published in a very short timeframe and the continuous flow of new data leads to difficulties in summarising best evidence for clinical practice for severe COVID-19 treatment.

What does this study add?

- Multifactorial pathophysiological mechanisms, including immune and non-immune cell abnormalities and loss of endothelial homeostasis, play a key role in COVID-19.

- These are first EULAR-endorsed 'points to consider' (PtC) providing a framework to optimise the use of immunomodulatory therapies for the care of people with SARS CoV-2 infection.

- This article highlights areas of therapeutic success but also areas of uncertainty (eg, timing/add-in approach for glucocorticoids in combination with other immunomodulatory agents) that require to be addressed by further research and barriers to overcome in order to develop new therapeutic strategies for SARSCoV-2 infection.

from asymptomatic or mild disease associated with an uneventful recovery to severe disease leading to acute respiratory distress syndrome (ARDS), multiorgan failure and death. ${ }^{1}$ Immune mechanisms are critically involved in evolution of severe COVID19 , suggesting potential efficacy of immunomodulatory treatments. ${ }^{23}$ 
Key messages

\section{How might this impact on clinical practice?}

- It is anticipated that clinicians will use these PtC as a basis for the optimal management of people with SARS-CoV-2 infection and for further strategies for optimal therapy.

- These PtC will also support relevant stakeholders when evaluating new immunomodulatory therapeutic approaches to treat SARS-CoV-2 infection.

Since rheumatologists are at the vanguard of immunomodulatory therapy utilisation, they are well placed to provide insights in their usage, safety profile and timing towards potential mitigation of the severe inflammatory reactions associated with SARS-CoV-2 infection. Although an extremely large volume of publication is already available, most of the research is descriptive and definitive randomised controlled trials (RCTs) remains scarce. In this rapidly evolving landscape, and with the need to optimally define therapeutics solutions, it is considerably important to provide the medical and scientific community with guidance using the best available evidence. This is especially the case since antiviral agents have thus far failed to show a significant improvement of survival in COVID-19. ${ }^{4-6}$ The aim of this project was to elaborate EULAR points to consider (PtCs) on COVID-19 pathophysiology and immunomodulatory therapies from the rheumatology perspective through a systematic literature review (SLR)-based approach.

\section{METHODS}

After approval by the EULAR Executive Committee, the convenors (XM and DGM) and the methodologist (PMM) led a multidisciplinary Task Force guided by the 2014 updated EULAR standardised operating procedures. ${ }^{7}$ The Task Force consisted of 24 members from 8 different countries, including rheumatologists, translational immunologists (two of them also representing the Emerging EULAR Network), haematologists, paediatric rheumatologists, a methodologist, one health professional and one patient representative. Four virtual meetings of the Task Force were held, one inJuly 2020 and three in November 2020. Two fellows (AA and AN), guided by the methodologist, performed an SLR, retrieving individual studies on pathophysiology of SARS-CoV-2 infection and its management with immunomodulatory therapies. Given the fast-paced publication of articles on treatment with potential impact on the formulation of PtCs, the last SLR update was performed on 11 December 2020. The SLR is published separately, however, it forms an integral part of the project.

Based on the presented evidence and expert opinion, and following a process of iterative discussion, the overarching principles (OPs) and PtCs were developed. According to EULAR guidance, the steering group (convenors, methodologist and fellows) prepared draft-statements based on the evidence from the SLRs and each statement was presented, discussed and voted on (informal voting). The statements were accepted if more than $75 \%$ of the Task Force approved the wording in the first round. If this was not reached, further discussion ensued and wording was refined. At least a $67 \%$ approval rate was required in the second voting round and more than $50 \%$ in the third round. ${ }^{8}$ The level of evidence (LoE) supporting each statement was assigned according to the Oxford Centre for Evidence Based Medicine 2011 LoE. $^{9}$ Finally, each Task Force member anonymously indicated their level of agreement with each $\mathrm{PtC}$ online (numerical rating scale ranging from $0=$ 'completely disagree' to $10=$ 'completely agree'). Based on the heterogeneity of studies gathered by the SLR and on the paucity of high-quality evidence (namely RCTs) despite the large amount of published articles, a research agenda was formulated. The final manuscript was reviewed and approved by all Task Force members, followed by ratification by the EULAR Executive Committee.

\section{RESULTS}

Two OP and 14 PtCs were formulated and encompass the pathophysiology of SARS-CoV-2 infection and its treatment with immunomodulatory agents (table 1 ). The $\mathrm{PtC}$ are intended to provide insights in pathogenesis and guidance on therapeutic aspects, and the target users are healthcare providers taking care of patients infected with SARS-CoV-2 as well as patients and policy-makers.

\section{Overarching principles}

OP-A: the phenotype of SARS-CoV-2 infection is heterogeneous, ranging from asymptomatic to fatal disease due to multiorgan damage

The Task Force recognised the importance of outlining this concept as OP to set the scenario for the PtCs. A heterogeneous clinical spectrum is a hallmark of SARS-CoV-2 infection and a challenge for the clinicians. Patients with severe manifestations, severe systemic inflammation and ARDS remain difficult to treat leading to a negative outcome in a high number of cases.

OP-B: SARS-CoV-2 infection may need different treatment approaches, including antiviral, oxygen therapy, anti-coagulation and/or immunomodulatory treatment at different stages of the disease

An increasing number of articles outlined the complexity of SARS-CoV-2 pathophysiology and recommendations issued by the WHO and other key stakeholders ${ }^{10-12}$ reflect the necessity to combine supportive therapy along with agents with different mechanisms of action, for example, antiviral treatment, oxygen therapy, anticoagulation and the inflammatory response along with other key mechanism involved in the process. However, it is not yet clear when and how to combine these various approaches (eg, combination vs sequential therapy).

\section{PtCs: pathophysiology of COVID-19}

PtC-1: genetically determined differences including, but not limited to, immune gene pathways may contribute to the variable immune response to SARS-CoV-2 and ultimately impact on the disease prognosis (LoE 3/4)

Genetic differences may contribute to the clinical heterogeneity of SARS-CoV-2 infection with the type 1 interferon (IFN) pathway emerging as the strongest genetic association in both genome wide association studies (GWAS) and from Mendelian genetic studies of critically ill patients. ${ }^{13}$ A GWAS study also reported associations with immune response genes such as $\mathrm{C}-\mathrm{C}$ Motif Chemokine Receptor 9 and C-X-C Motif Chemokine Receptor 6, and severe COVID-19. ${ }^{14}$ The same study reported higher risk of severe disease in people with blood group A. ${ }^{14}$ Whole-exome sequencing reported variants of genes involved in IFN mediated immune response in a subset of patients with severe COVID-19 and patients showing these variants had hampered IFN immunity in vivo and in vitro. ${ }^{15-18}$ Data concerning (HLA) haplotypes are still conflicting. ${ }^{14} 19$ To note, low allelic variability of the ACE-2, whose binding by the SARS-CoV-2 spike protein facilitates cellular entry of the virus, was also observed 
Table 1 Overarching principles and points to consider on COVID-19 pathophysiology and immunomodulatory treatment from the rheumatology perspective, with levels of evidence (LoE) and levels of agreement (LoA)

\begin{tabular}{|c|c|}
\hline Overarching principles & $\begin{array}{l}\text { LoA mean }(S D) ; \\
\% \text { of votes } \geq 8 / 10\end{array}$ \\
\hline A. The phenotype of SARS-CoV-2 infection is heterogeneous ranging from asymptomatic to lethal disease due to multiorgan damage. & $9.92(0.3) ; 100$ \\
\hline $\begin{array}{l}\text { B. SARS-CoV-2 infection may need different treatment approaches, including antiviral, oxygen therapy, anticoagulation and/or immunomodulatory treatment } \\
\text { at different stages of the disease. }\end{array}$ & $9.92(0.3) ; 100$ \\
\hline \multicolumn{2}{|l|}{ Points to consider } \\
\hline \multicolumn{2}{|l|}{ COVID-19 pathophysiology } \\
\hline $\begin{array}{l}\text { 1. Genetically determined differences including, but not limited to, immune gene pathways may contribute to the variable immune response to SARS-CoV-2 } \\
\text { and ultimately impact on the disease prognosis (LoE } 3 / 4) \text {. }\end{array}$ & $9.25(1.2) ; 100$ \\
\hline $\begin{array}{l}\text { 2. Cellular (LoE 3/4) and humoral ( } L O E \text { 3) immune responses against SARS-CoV-2 vary across individuals, infection course and disease spectrum, but there is } \\
\text { insufficient evidence to associate these directly with outcomes. }\end{array}$ & $8.71(1.1) ; 83$ \\
\hline 3. Levels of many proinflammatory cytokines, especially serum IL-6, are elevated in COVID-19 and could be associated with outcome (LoE 3/4). & 8.79 (1.0): 96 \\
\hline $\begin{array}{l}\text { 4. Hyperactivation of platelets, the complement system, endothelial damage and loss of endothelial homeostasis are pathophysiological mechanisms } \\
\text { facilitating hypercoagulability and thrombosis during SARS-CoV-2 infection (LoE 4). }\end{array}$ & $9.0(1.1) ; 92$ \\
\hline $\begin{array}{l}\text { 5. Multiparameter algorithms including neutrophil-to-lymphocyte ratio and acute phase reactants (eg, } \mathrm{C} \text { reactive protein, ferritin) may be helpful to predict } \\
\text { survival, mortality or disease progression and severity (LoE 4). }\end{array}$ & $8.88(1.3) ; 88$ \\
\hline $\begin{array}{l}\text { 6. Primary infection with SARS-CoV-2 in children is largely a benign event. However, a small number of children develop a multisystem inflammatory } \\
\text { syndrome which may reflect distinct pathophysiological mechanisms compared with adults (LoE } 4 / 5 \text { ). }\end{array}$ & $9.38(0.9) ; 96$ \\
\hline \multicolumn{2}{|l|}{ Immunomodulatory therapy } \\
\hline 7. In non-hospitalised patients with SARS-CoV-2 infection there is currently no evidence to support the initiation of immunomodulatory therapy (LoE 2/3/4). & $9.58(1.0) ; 96$ \\
\hline $\begin{array}{l}\text { 8. In hospitalised patients with SARS-CoV-2 infection that do not need oxygen therapy there is currently no evidence to support the initiation of } \\
\text { immunomodulatory therapy to treat their COVID-19 (LoE } 2 / 3 / 4 \text { ). }\end{array}$ & $9.04(1.6) ; 88$ \\
\hline $\begin{array}{l}\text { 9. Hydroxychloroquine should be avoided for treating any stage of SARS-CoV-2 infection since it does not provide any additional benefit to the standard of } \\
\text { care, and could worsen the prognosis in more severe patients particularly if co-prescribed with azithromycin (LoE 2). }\end{array}$ & $9.75(0.5) ; 100$ \\
\hline $\begin{array}{l}\text { 10. In patients with COVID-19 requiring supplemental oxygen, non-invasive or mechanical ventilation, systemic glucocorticoids should be used since they } \\
\text { can decrease mortality; most evidence concerns the use of dexamethasone (LoE } 2 / 3 \text { ). }\end{array}$ & $9.67(0.7) ; 100$ \\
\hline $\begin{array}{l}\text { 11. An evolving RCT landscape cannot yet allow formal recommendation of the routine use of tocilizumab in patients with COVID-19 requiring oxygen } \\
\text { therapy, non-invasive or invasive ventilation (LoE 2). }\end{array}$ & 8.79 (1.2): 83 \\
\hline 12. In COVID-19 there is no robust evidence to support the use of anakinra at any disease stage (LoE 2/4). & $9.38(1.0) ; 96$ \\
\hline $\begin{array}{l}\text { 13. In patients with COVID-19 requiring non-invasive ventilation or high-flow oxygen, the combination of remdesivir plus baricitinib could be considered } \\
\text { since it can decrease time to recovery and accelerate improvement in clinical status (LoE 2). }\end{array}$ & $8.19(2.2) ; 88$ \\
\hline $\begin{array}{l}\text { 14. In COVID-19 there is currently insufficient evidence to recommend the use of other immunomodulators, including ruxolitinib, IVIg, convalescent plasma } \\
\text { therapy except in Ig-deficient patients, interferon kappa, interferon beta, leflunomide, colchicine (LoE 2), sarilumab, lenzilumab, eculizumab, cyclosporine, } \\
\text { interferon alpha (LOE 3), canakinumab (LOE 4). }\end{array}$ & $9.42(0.9) ; 96$ \\
\hline
\end{tabular}

IL-6, interleukin 6; IVlg, intravenous Ig; RCT, randomised controlled trial.

in infected patients compared with normal subjects but was not associated with disease severity. ${ }^{20-22}$

PtC-2: cellular (LoE 3/4) and humoural (LoE 3) immune responses against $S A R S-C o V-2$ vary across individuals, infection course and disease spectrum, but there is insufficient evidence to associate these directly with outcomes

The activation of phagocytes, antigen-sensitised T cells and B-cells have been extensively studied in SARS-CoV-2 infection. It is worth noting, however, that most studies assessed individual cell populations through extremely variable methods (ie, flow cytometry gating and sub-population definition) and measured specific anti-SARS-CoV-2 immunoglobulins with home-made assays using different viral proteins as substrate, thereby preventing definitive conclusions. Among the few studies using an unsupervised clustering approach through mass cytometry and other high throughput sequencing techniques (single cell RNA sequencing, Cellular Indexing of Transcriptomes and Epitopes, Assay for Transposase-Accessible Chromatin, among others) several cell populations where reported to be consistently altered. The lymphoid compartment disruption was mostly characterised by a reduction in CD4 and CD 8 positive lymphocytes in patients with SARS-CoV-2 infection compared with healthy controls but also in patients displaying a severe disease vs milder phenotypes. $^{23-32}$ In addition, this subset of patients displayed a higher amount of polyfunctional $\mathrm{T}$ cells along with an exhausted CD8 + phenotype as highlighted by a high PD-1+ expression. ${ }^{232529}$ An alteration of cytotoxicity, as suggested by a reduction in granzymes A, B and perforin expression by CD8 + cells, was reported by one study. ${ }^{23}$ Natural killer and B-lymphocytes pools were also reduced in patients with SARS-CoV-2 infection when compared with healthy donors regardless of disease severity. ${ }^{25} 27$ In terms of myeloid response, an increase of classical CD14+ monocytes pool was consistently reported, along with a significant reduction of HLA-DR expression; as opposed to a reduction of dendritic cells in both mild and severe patients compared with healthy controls. ${ }^{2326-2833-36}$ In addition, the pool of immature neutrophils (pre and pro-neutrophils) was reported to be increased. ${ }^{35} 36$

With regard to the humoural response, a few studies used standardised, although based on different viral antigens, commercially available ELISA or chemiluminescence immunoassays. ${ }^{37-41}$ The authors reported appearance of anti-SARS-CoV-2 IgM within the first 2 weeks after the onset of symptoms but the fate of these antibodies is still controversial with studies reporting variable kinetics up to weeks 4-5 after the onset of symptoms. Conversely, anti-SARS-CoV-2 IgG variably appear either together with IgM in week $1^{4041}$ or in weeks $2-3^{37} 38$ being still detectable up to week $4,{ }^{38}$ week $6^{3740}$ or week $8 .{ }^{41}$ Data on the kinetics of neutralising antibodies are even more controversial. Additionally, results on other isotypes ( $\operatorname{IgA}$ ) are scarce, although potentially relevant for the clinical setting.

PtC-3: levels of many proinflammatory cytokines, especially serum interleukin-6, are elevated in COVID-19 and could be associated with outcome (LoE 3/4)

The cytokine expression profile during the course of SARS-CoV-2 infection has already been extensively studied. Since the level of proinflammatory cytokines is elevated in some stages of the disease, but not at very high levels, the group considered 
that the term severe systemic inflammatory state seems more appropriate than the frequently used 'cytokine storm'. ${ }^{42}$ Of interest, while most studies used a targeted approach towards one cytokine or one family of cytokines subsequently leading to bias, only a few used multiparametric assays allowing the multivariable assessment of the expression of several cytokines at the same time. Among these, several studies reported a consistent elevation of interleukin (IL)-6, IL-1 beta and tumor necrosis factor along with Th1 associated cytokines (IFN-gamma, IL-18) in COVID-19 patients compared with healthy donors but the magnitude of cytokine elevations was greater in patients displaying more severe disease phenotypes compared with mild or moderate diseases. ${ }^{2833} 43-45$

These results are concordant with the large amount of studies correlating IL-6 levels with disease severity and negative outcomes. ${ }^{46}$ In addition to these, anti-inflammatory cytokines and a broad range of chemokines and growth factors expression profiles were inconsistently reported to be disrupted to a variable extent according to disease severity and outcomes but these results were not felt consistent enough by the TF to lead to the formulation of a PtC. ${ }^{47}$ However, the extent of cytokinaemia in cases of severe and critical COVID-19 is less than that seen in other disorders associated with elevated cytokine production, such as chimeric antigen receptor T-cell-induced cytokine release syndrome and non-COVID-19 ARDS. ${ }^{42} 48$

PtC-4: hyperactivation of platelets, the complement system, endothelial damage and loss of endothelial homeostasis are pathophysiological mechanisms facilitating hypercoagulability and thrombosis during SARS-CoV-2 infection (LoE 4)

A large number of studies described an increased prevalence of thrombosis and thromboembolic disease in patients with SARS-CoV-2 infection. ${ }^{49}$ Controversy still exists as to whether hypercoagulability in SARS-CoV-2 infection may be immune driven or immunothrombotic in nature or may be linked to direct viral endothelitis (or both). Platelet aggregation and activation, as expected in the context of endothelial injury and immune activation were observed and were more pronounced in severe COVID-19..$^{50-60}$

PtC-5: multiparameter algorithms including neutrophilto-lymphocyte ratio and acute phase reactants (eg, $C$ reactive protein, ferritin) may be helpful to predict survival, mortality or disease progression and severity (LoE 4)

In order to help clinicians in disease management, several studies aiming at creating algorithms for prediction of disease progression, severity or outcomes have been published. ${ }^{61-73}$ Among them, a few have used robust statistical approaches using uni- and multivariate analysis, followed by logistic regression or least absolute shrinkage and selection operator. Most of them included various parameters from clinical data (demographics, comorbidities, symptoms), biological results (acute phase reactants such as C reactive protein, ferritin, procalcitonin, calprotectin; coagulation markers such as platelets, D-dimer, prothrombin time; immune cell count such as neutrophils, lymphocytes or neutrophil-to-lymphocyte ratio) or imaging results (chest CT).

The relevance of combining different parameters for prognostic purposes in further underscored by the recent release of predictive criteria for COVID-19 severe systemic inflammatory state. $^{74}$

PtC-6: primary infection with SARS-CoV-2 in children is largely a benign event. However, a small number of children develop a multisystem inflammatory syndrome which may reflect distinct pathophysiological mechanisms compared with adults (LoE 4/5)
Epidemiological data pertaining to SARS-CoV-2 infection in children are rather homogeneous describing mild to moderate symptoms in most cases. ${ }^{75}$ However, from the end of April 2020, a number of alerts have been raised in several countries about children with proven SARS-CoV-2 infection developing a multisystem inflammatory syndrome (MIS-C). ${ }^{76}$ More recently reports of MIS-C in young adults have been published. ${ }^{77}$ Studies comparing clinical and serological features of children and adults with SARS-CoV-2 infection are scarce and included small cohorts of patients hence they do not allow to draw definitive conclusions on similarities and differences of SARS-CoV-2 infection across the lifespan. ${ }^{78}$ However, the discussion on this $\mathrm{PtC}$ was led by the paediatric rheumatologists and resulted in an agreement to include expert opinion given the paucity of data from the SLR. In particular, the Task Force supported the hypothesis that this clinical heterogeneity may reflect distinct pathophysiological mechanisms, not yet fully elucidated, that occur in children and in adults with SARS-CoV-2 infection.

\section{PtCs: immunomodulatory treatment}

PtC-7: in non-hospitalised patients with SARS-CoV-2 infection there is currently no evidence to support the initiation of immunomodulatory therapy (LoE 2/3/4)

The literature assessing the efficacy of immunomodulatory therapies on reducing time to an undetectable viral load, reducing the duration of clinical symptoms or preventing clinical deterioration in patients presenting with mild or moderate forms including mild hypoxaemia not requiring hospitalisation remains scarce since most of the clinical research has included more severe patients. However, it is worth noting that among the few studies available, none provided evidence of treatment efficacy in this specific population. More specifically, none of the RCTs assessing the efficacy of hydroxychloroquine (HCQ) in non-hospitalised patients reported positive outcomes. ${ }^{7980} \mathrm{It}$ is possible that this phase of SARS-CoV-2 infection requires more efficient anti-viral drugs than immunomodulatory drugs.

PtC-8: In hospitalised patients with SARS-CoV-2 infection that do not need oxygen therapy there is currently no evidence to support the initiation of immunomodulatory therapy (LoE 2/3/4)

A large number of studies on hospitalised patients with SARS-CoV-2 infection and various disease severity investigated immunomodulatory therapy. However, none of the investigated compounds except leflunomide in one small RCT, ${ }^{81}$ specifically demonstrated to be effective if added to standard of care (SOC) in hospitalised patients with mild-to-moderate disease not requiring oxygen therapy compared with SOC only. Of note, in the large RECOVERY RCT, analysis of the subgroup of patients without oxygen support showed a possible (not statistically significant) deleterious effect of dexamethasone (DEX) on mortality: OR $1.22,95 \%$ CI 0.93 to $1.61, \mathrm{p}=0.14 .^{82}$ Likewise, HCQ was clearly ineffective in three RCTs including patients with mild to moderate disease and it even showed a negative safety profile. ${ }^{79} 80$ Due to the lack of efficacy and the safety concerns also in patients with severe COVID-19, and the historical debate about the role of HCQ in SARS-CoV-2 infection, with HCQ shortages being reported during the SARS-CoV-2 pandemic, the group decided to elaborate a separate $\mathrm{PtC}$ for this drug.

PtC-9: HCQ should be avoided for treating any stage of SARS-CoV-2 infection since it does not provide any additional benefit to the SOC, and could worsen the prognosis in more severe patients particularly if coprescribed with azithromycin (LoE 2) 
In the early phases of the SARS-CoV-2 pandemic, HCQ was extensively used for the management of infected patients due to its known antiviral and immunomodulatory effects. ${ }^{83}$ However, the initial promising data from prospective/retrospective studies have not been confirmed by RCTs. Data from eight RCTs on HCQ in patients with SARS-CoV-2 infection and different degrees of COVID-19 severity consistently showed that the addition of this compound to SOC did not provide any benefit at any stage of the disease. ${ }^{79} 8084-89$ Importantly, the RECOVERY study reported that for some outcomes such as progression to invasive mechanical ventilation (IMV) or death, and probability to be discharged alive, the addition of HCQ to SOC led to even worse outcomes compared with SOC alone. Furthermore, safety concerns have been raised since patients treated with HCC +SOC displayed a higher rate of adverse events compared with SOC only at any disease stage, mainly if associated with azithromycin. For example, HCQ-treated patients with severe COVID-19 displayed prolongation of the corrected QT interval, elevation of liver-enzymes and higher risk of death from nonSARS-CoV-2 cardiac causes. ${ }^{86} 87$

PtC-10: in patients with COVID-19 requiring supplemental oxygen, non-IMV, systemic glucocorticoids should be used since they can decrease mortality; most evidence concerns the use of DEX (LoE 2/3)

This PtC was elaborated based on the results of 6 RCTs on patients with severe COVID-19. Two trials investigated DEX, ${ }^{8290}$ two investigated methylprednisolone (MTP), ${ }^{91} 92$ and two investigated hydrocortisone. ${ }^{9394}$ While the overall analysis of patients yielded conflicting results for the majority of outcomes, the evidence on mortality from subgroup analysis was striking and revealed the beneficial effect of DEX and MTP in specific subgroups of patients. The RECOVERY trial demonstrated that the addition of DEX to SOC reduced mortality only in patients requiring respiratory support. ${ }^{82}$ The addition of MTP to SOC reduced mortality only in patients aged 60 or over by mechanisms potentially including rescue of immunosenescence. ${ }^{91}$ The group agreed that the evidence was more consistent for DEX rather than for MTP. Based on this data, DEX has been included in most of the recommendations of treatment for critical intensive care unit (ICU) patients and for mild-to-moderate pneumonia needing oxygen in medical wards. Of note, in the latter population, there is no study to decipher the effect of glucocorticoids between the patients requiring a low rate of oxygen $1-2 \mathrm{~L} / \mathrm{min}$ ) and those requiring higher rate $(3-15 \mathrm{~L} / \mathrm{min})$. It is an important point since the pathophysiology might be different in both groups.

PtC-11: an evolving RCT landscape cannot yet allow formal recommendation of the routine use of tocilizumab in patients with COVID-19 requiring oxygen therapy, non-invasive or invasive ventilation ( $L O E$ 2)

The SLR retrieved 104 articles evaluating tocilizumab (TCZ) in SARS-CoV-2 infection and only three were RCTs. ${ }^{95-97} \mathrm{~A}$ scenario similar to that reported above for HCQ occurred for TCZ. Based on the evidence of the severe systemic inflammation at some stages of the disease, where IL- 6 is perceived to be 'a leading actor' and on results from early studies with low LoE, many of which lacking a control group, prompted initial widespread TCZ use. Two published RCT ${ }^{9697}$ were negative but probably included mild patients, some of them not requiring oxygen support since day-28 mortality was low: $4.9 \%$ and $2.4 \%$, respectively. The third published study (CORIMUNO-19) ${ }^{95}$ focusing on patients requiring at least $3 \mathrm{~L} / \mathrm{min}$ oxygen but not in ICU showed a 33\% reduction of non-invasive or invasive ventilation (NIV) or death at day 14 , but day-28 mortality was not different between groups (around 12\%). Of note, two studies in the preprint phase, the first one (EMPACTA) with the same inclusion criteria as CORIMUNO-19), and positive with the same composite primary outcome, the second one (COVACTA) negative for its primary outcome, but with positive results in the post hoc analysis of patients not in ICU. Lastly, in the patients recently admitted in ICU within 2 days, the REMAP-CAP study was prematurely stopped because of positive results on survival with $\mathrm{TCZ}^{98}$ and an emulated trial in the same population found an improvement of survival. ${ }^{99}$ Interestingly, safety of TCZ was good in all these studies with in some of them a decrease of the rate of serious infections in the TCZ arm versus placebo or usual care arm. To summarise, despite the encouraging signals in some subgroups, it remains impossible at the date of this paper to draw definitive conclusions and to formulate a $\mathrm{PtC}$ either recommending or discouraging the use of TCZ in severe COVID-19. Meta-analysis on aggregated or individual patient data and further clinical trials are warranted and results from these trials will inform about the potential benefit of TCZ, in selected patients. Observational studies suggested benefit of glucocorticoids in combination with TCZ, but RCTs are needed for the evaluation of TCZ in addition to DEX/MTP vs DEX/ MTP alone. This will be an important step for determining the possible place of this drug as an 'add on therapy' in glucocorticoid non-responsive cases.

PtC-12: in COVID-19 there is no robust evidence to support the use of anakinra at any disease stage (LoE 2/4)

At present, the only RCT available on anakinra in SARS-CoV-2 infection was conducted by the CORIMUNO-19 Collaborative group in mild-to-moderate COVID-19 pneumonia requiring at least $3 \mathrm{~L} / \mathrm{min}$ oxygen but not receiving NIMV or IMV ventilation at randomisation. The study was stopped early following the recommendation of the data and safety monitoring committee since no beneficial effect was observed. ${ }^{100}$ As far as moderateto-severe COVID-19 is concerned, conflicting results have been reported from three retrospective controlled studies. ${ }^{101-103}$

PtC-13: in patients with COVID-19 requiring NIV or high-flow oxygen, the combination of remdesivir plus baricitinib could be considered since it can decrease time to recovery and accelerate improvement in clinical status (LoE 2)

At present, the only RCT available on baricitinib in SARS-CoV-2 infection compared remdesevir + baricitinib versus remdesevir + placebo. ${ }^{104}$ Patients receiving baricitinib had a median time to recovery of 7 days, as compared with 8 days with control (rate ratio for recovery, $1.16 ; 95 \%$ CI 1.01 to $1.32 ; p=0.03$ ), except in the subgroup of patients with a baseline NIV (including high flow oxygen) in whom median time to recovery was 10 days with the combination, as compared with 18 days with control (rate ratio for recovery, $1.51 ; 95 \%$ CI 1.10 to 2.08). In light of these data, the main issue that emerged during the discussion pertained to the target population in which to recommend baricitinib + remdesivir. The majority of Task Force members agreed to suggest baricitinib + remdesivir only in the subgroup of patients with a baseline NIV since the small difference in time to recovery observed in the overall cohort was felt clinically not pertinent, although statistically significant.

PtC-14: in COVID-19 there is currently insufficient evidence to recommend the use of other immunomodulators, including ruxolitinib, IVIg, convalescent plasma therapy except in Ig-deficient patients, IFN kappa, IFN beta, leflunomide, colchicine (LoE 2), sarilumab, lenzilumab, eculizumab, cyclosporine, IFN alpha (LoE 3), canakinumab (LoE 4)

Various other immunomodulatory compounds have been investigated in SARS-CoV-2 infection. However, the available 
evidence to date is either too scarce/conflicting, although from RCTs, as in the case of ruxolitinib, IFN beta, IFN kappa, IVIg and convalescent plasma or with too low LoE/conflicting results as in the case of sarilumab, lenzilumab, eculizumab, IFN alpha, tumour necrosis factor alpha inhibitors and canakinumab (LoE $3 / 4)$. Hence, no recommendation either in favour or against the use of these compounds in SARS-CoV-2 infection could be formulated. The group did not comment on drugs for which published literature was not available, although being in the pipeline.

\section{DISCUSSION}

These are the first EULAR-endorsed PtCs on pathophysiology and immunomodulatory therapy of SARS-CoV-2 infection. Their aim is to serve as a reference for clinicians involved in the care of people with SARS-CoV-2 infection and the use of immunomodulatory therapy as seen from the translational rheumatology perspective. They have been proposed by a rheumatology/multidisciplinary team. Since rheumatologists are the experts on inflammatory systemic diseases and on the use of immunomodulatory therapy, they should be involved in mechanistic/pathophysiological studies and in the design, conduct and interpretation of trials with immunomodulatory therapies in COVID-19.

With the OPs, we recognised that the management of SARS-CoV-2 infection, with its heterogeneous nature, requires a multifaceted approach considering viral replication, hypercoagulability and the inflammatory response, the latter of which was our specific focus. In this regard, these PtCs focus on pathophysiology and immunomodulatory treatment and the discussion leading to formulation of these PtCs was based not only on the evidence obtained by the SLR but also on the opinion of experts of inflammation and immunomodulatory therapy.

These PtCs in no way attempt to undermine local regulations or guidelines released by overarching institutions such as the WHO. Rather, they seek to provide recommendations of good practice integrating evidence-based medicine and expert opinion, which can help clinicians analysing their own therapeutic strategy and inspire changes where appropriate. It is important to emphasise that our findings do not apply to people living with rheumatic and musculoskeletal diseases (RMDs) receiving immunomodulatory treatments where a dedicated EULAR Task Force has already developed provisional recommendations for the management of people with RMDs in the context of SARS-CoV-2 pandemic. ${ }^{105}$

One major pitfall encountered in the development of these PtCs was the extremely large bulk of literature that challenged its appraisal including a surprisingly high number of articles lacking original data, thus being uninformative to the discussion. Interestingly, the narrative around 'cytokine storm' and its specific therapy at the start of pandemic lacked evidence so was not included in the OP or PTC in the present recommendations. The rapidly evolving COVID-19 landscape along with the ongoing release of new studies represents a major challenge, since recommendations should only be elaborated on existing published peer-reviewed literature and following robust methodological procedures. To overcome these issues, we performed hand-search work throughout the entire SLR process and TF meetings, and based these PtCs on a very recent literature update. Based on the SLR results and the inputs from the group, a research agenda was also outlined (box 1).
Box 1 Research agenda

Pathophysiology of SARS-CoV-2 infection

- To explore in depth the abnormalities of the immune system with mechanistic studies.

- To better characterise the associations between immune alterations and SARS-CoV2 infection stages.

- To elucidate the actual role of type I and type II interferons across the different stages and their potential use as therapeutic agents.

- To elucidate the principles of effective immunity in agingageing individuals, namely in the context of SARS-CoV-2 infection.

- To perform studies with unsupervised multiparametric approaches aimed at identifying reliable biomarkers for prediction of disease outcomes and response to immunomodulatory treatment.

- To perform genome-wide association studies in patients with SARS-CoV-2 infection and various degrees of COVID-19 severity.

- To perform comparative studies including large cohorts of adult and paediatric patients with different degree of severe systemic inflammation and disease severity, investigating not only clinical and serological assessment but also mechanistic aspects of the immune response.

- To explore the possibility that SARS-CoV-2 infection may induce autoimmune diseases and/or flares of known conditions.

- To better define subsets of severe COVID-19 patients with severe systemic inflammation and the possible application of immunomodulatory therapy in this subset.

\section{Immunomodulatory treatment of SARS-CoV-2 infection}

- To define harmonised inclusion criteria and outcomes for both randomised controlled trials investigating immunomodulatory drugs and translational studies.

- To conduct randomised controlled trials investigating immunomodulatory drugs that to date have been explored only in studies with lower level of evidence.

- To conduct randomised controlled trials comparing the efficacy and safety of combining glucocorticoids and other immunomodulatory agents (eg, anticytokines) versus glucocorticoids alone.

In conclusion, these EULAR PtCs provide relevant guidance on the pathophysiology of SARS-COV-2 infection, especially immunomodulatory therapy utilisation from the rheumatology perspective. Indeed, the pathophysiology with an initial viral alveolitis and subsequent immunothrombosis and available evidence points towards a central role for immunomodulatory therapy, especially glucocorticoids, in improving disease survival in severe COVID-19. These first EULAR PtCs are intended to evolve and should be updated in response to the rapid increase of knowledge about the disease and the forthcoming vaccines.

\section{Author affiliations}

${ }^{1}$ Rheumatology Unit, Department of Medicine, University of Perugia, Perugia, Italy ${ }^{2}$ Institute of Infection, Immunity and Inflammation, College of Medical Veterinary and Life Sciences, University of Glasgow, Glasgow, UK

${ }^{3}$ Centre for Rheumatology \& Department of Neuromuscular Diseases, University College London, London, UK

${ }^{4}$ National Institute for Health Research (NIHR) Biomedical Research Centre (BRC), University College London Hospitals NHS Foundation Trust, London, UK 
${ }^{5}$ Department of Rheumatology, Northwick Park Hospital, London North West University Healthcare NHS Trust, London, UK

${ }^{6}$ Oslo, Norway

${ }^{7}$ Department of Rheumatology and Clinical Immunology, Charité -

Universitätsmedizin Berlin, Freie Universität und Humboldt-Universität Berlin, Berlin, Germany

${ }^{8}$ Department of Medicine, ASL 1 Avezzano-Sulmona-L'Aquila, Internal Medicine and Nephrology Unit, Department of Life, Health \& Environmental Sciences, University of L'Aquila, L'Aquila, Italy

The Leeds Institute of Rheumatic and Musculoskeletal Medicine, University of Leeds, Leeds, UK

${ }^{10}$ Rheumatology and Clinical Immunology Unit, University of Rome "Campus

Biomedico" School of Medicine Rome, Rome, Italy

${ }^{11}$ Department of Haematology, Hôpital Necker, Assistance Publique - Hôpitaux de

Paris, Paris, France

${ }^{12}$ INSERM UMR1183, Institut Imagine, Université de Paris, Paris, France

${ }^{13}$ Translational and Clinical Research Institute, Newcastle University and

Musculoskeletal Unit, Newcastle Hospitals NHS Foundation Trust, Newcastle upon Tyne, UK

${ }^{14}$ Service de Rhumatologie Pédiatrique, Centre de Référence des Maladies AutoInflammatoires de l'enfant, Hôpital Bicêtre, AP HP, Université Paris Sud, Bicètre, France

${ }^{15}$ Department of Rheumatology, Zuyderland Medical Centre Heerlen, Heerlen, The Netherlands

${ }^{16}$ Experimental Laboratory of Immunological and Rheumatologic Researches, Istituto Auxologico Italiano, IRCCS, Milan, Italy

${ }^{17}$ Department of Medicine, Rheumatology Clinic, University of Udine, ASUFC Udine, Udine, Italy

${ }^{18}$ University Hospitals Bristol NHS Foundation Trust, Bristol, UK

${ }^{19}$ University of Bristol Translational Health Sciences, Bristol, UK

${ }^{20}$ Department of Autoimmune Diseases, ICMiD, Laboratory of Autoimmune Diseases Josep Font, IDIBAPS-CELLEX, Department of Autoimmune Diseases, ICMiD, University of Barcelona, Hospital Clínic, Barcelona, Spain

${ }^{21}$ Department of Functional Biology, Immunology Area, Faculty of Medicine, University of Oviedo, Instituto de Investigación Sanitaria del Principado de Asturias (ISPA), Oviedo, Spain

${ }^{22}$ Division of Rheumatology and Clinical Immunology, Department of Internal Medicine IV, Ludwig-Maximilians University of Munich, Munchen, Germany ${ }^{23}$ Section for Outcomes Research, Center for Medical Statistics, Informatics, and Intelligent Systems, Medical University of Vienna and Ludwig Boltzmann Institute for Arthritis and Rehabilitation, Wien, Austria

${ }^{24}$ Department of Rheumatology and Clinical Immunology, Amsterdam Rheumatology and Immunology Center, Amsterdam University Medical Centres, Amsterdam, The Netherlands

${ }^{25}$ Department of Internal Medicine, Cochin University Hospital, Paris, France; National Referral Centre for Systemic and Autoimmune Diseases, University Paris Descartes, Sorbonne Paris Cité, Paris, France

${ }^{26}$ Assistance Publique-Hôpitaux de Paris, Hôpital Bicêtre, INSERM UMR1184, Department of Rheumatology, Université Paris-Saclay, Le Kremlin Bicêtre, France

Twitter Pedro M Machado @pedrommcmachado and John D Isaacs@ ProfJohnlsaacs

Contributors All authors contributed and finally approved the current manuscript. DGM and XM share last Authorship.

Funding This work was funded by European League Against Rheumatism (CLI122) PMM is supported by the National Institute for Health Research (NIHR) University College London Hospitals (UCLH) Biomedical Research Centre (BRC). JDI is a NIHR Senior Investigator and his work is supported by the NIHR Newcastle Biomedical Research Centre in Ageing and Long-Term Conditions, and the Research Into Inflammatory Arthritis Centre versus Arthritis. AVR is a member of the paediatric steering committee of RECOVERY, the steering committee of COVINTOC study and the steering committee of baricitinib in COVID-19.

Disclaimer The views expressed are those of the authors and not necessarily those of the (UK) National Health Service, NIHR or the Department of Health.

Competing interests $A A, A N, H B, F C, G D M, R G, C M-C$ and JRC have nothing to declare. PMM has received consulting and/or speaker's fees from Abbvie, BMS, Celgene, Eli Lilly, Janssen, MSD, Novartis, Orphazyme, Pfizer, Roche and UCB, all unrelated to this manuscript. GRB has received consulting and/or speaker's fees from Abbvie, Gilead, Lilly, Roche, Sanofi, Pfizer all unrelated to this manuscript. IK-P has received consulting and/or speaker's fees from Novartis, SOBI, Amgen, CHUGAI, Pfizer, LFB, Novimmune, Abbvie and PAtent for AIDAI score AVR has received speaker fees/Honoraria from Abbvie, Lilly, Roche, UCB, SOBI and Novartis all unrelated to this manuscript. DGM has received consulting and/or speaker's fees from Abbvie, BMS, Celgene, Eli Lilly, Janssen, MSD, Novartis, Pfizer, Roche and UCB, all unrelated to this manuscript. XM has received consulting and/or speaker's fees from BMS, Eli Lilly, Galapagos, Gilead, GSK, Janssen, Novartis, Pfizer, Servier and UCB, all unrelated to this manuscript.
Patient and public involvement Patients and/or the public were involved in the design, or conduct, or reporting, or dissemination plans of this research. Refer to the Methods section for further details.

Patient consent for publication Not required.

Provenance and peer review Not commissioned; externally peer reviewed.

Open access This is an open access article distributed in accordance with the Creative Commons Attribution Non Commercial (CC BY-NC 4.0) license, which permits others to distribute, remix, adapt, build upon this work non-commercially, and license their derivative works on different terms, provided the original work is properly cited, appropriate credit is given, any changes made indicated, and the use is non-commercial. See: http://creativecommons.org/licenses/by-nc/4.0/.

\section{ORCID iDs}

Alessia Alunno http://orcid.org/0000-0003-1105-5640

Aurélie Najm http://orcid.org/0000-0002-6008-503X

Pedro M Machado http://orcid.org/0000-0002-8411-7972

Francesco Carubbi http://orcid.org/0000-0003-1958-5136

John D Isaacs http://orcid.org/0000-0002-6103-7056

Isabelle Koné-Paut http://orcid.org/0000-0001-8939-5763

Pier Luigi Meroni http://orcid.org/0000-0002-3394-1451

Luca Quartuccio http://orcid.org/0000-0002-0134-6439

Hendrik Schulze-Koops http://orcid.org/0000-0002-1681-491X

Tanja A Stamm http://orcid.org/0000-0003-3073-7284

Benjamin Terrier http://orcid.org/0000-0001-6612-7336

\section{REFERENCES}

1 Wong CKH, Wong JYH, Tang EHM, et al. Clinical presentations, laboratory and radiological findings, and treatments for 11,028 COVID-19 patients: a systematic review and meta-analysis. Sci Rep 2020;10:19765

2 McGonagle D, Sharif K, O'Regan A, et al. The role of cytokines including interleukin- 6 in COVID-19 induced pneumonia and macrophage activation syndrome-like disease. Autoimmun Rev 2020;19:102537.

3 Magro C, Mulvey JJ, Berlin D, et al. Complement associated microvascular injury and thrombosis in the pathogenesis of severe COVID-19 infection: a report of five cases. Transl Res 2020;220:1-13.

4 Cao B, Wang Y, Wen D, et al. A trial of Lopinavir-Ritonavir in adults hospitalized with severe Covid-19. N Engl J Med 2020;382:1787-99.

5 Beigel JH, Tomashek KM, Dodd LE, et al. Remdesivir for the Treatment of Covid-19Final Report. N Engl J Med 2020;383:1813-26.

6 WHO Solidarity Trial Consortium. Repurposed antiviral drugs for Covid-19 — interim who solidarity trial results. N Engl J Med 2020.

7 van der Heijde D, Aletaha D, Carmona L, et al. 2014 update of the EULAR standardised operating procedures for EULAR-endorsed recommendations. Ann Rheum Dis 2015;74:8-13.

8 EULAR. EULAR Madrid EC meeting 2019. Available: https://www.eular.org/ myUploadData/files/voting_eular_recos_approved_exc_june_2019_web.pdf

9 CEBM. Centre for evidence-based medicine. Available: https://www.cebm.ox.ac.uk

10 WHO. Clinical management of COVID-19, 2020. Available: https://www.who.int/ publications//item/dinical-management-of-covid-19

$11 \mathrm{NIH}$. Coronavirus disease 2019 (COVID-19) treatment guidelines. Available: https:// www. covid19treatmentguidelines.nih.gov

12 IDSA. Infectious diseases Society of America guidelines on the treatment and management of patients with COVID-19, 2020. Available: https://www.idsociety. org/practice-guideline/covid-19-guideline-treatment-and-management/

13 Pairo-Castineira E, Clohisey S, Klaric L, et al. Genetic mechanisms of critical illness in Covid-19. Nature 2020. doi:10.1038/s41586-020-03065-y. [Epub ahead of print: 11 Dec 2020]

14 Severe Covid-19 GWAS Group, Ellinghaus D, Degenhardt F, et al. Genomewide association study of severe Covid-19 with respiratory failure. N Engl J Med 2020;383:1522-34.

15 Zhang Y, Qin L, Zhao Y, et al. Interferon-Induced transmembrane protein 3 genetic variant rs $12252-C$ associated with disease severity in coronavirus disease 2019. J Infect Dis 2020;222:34-7.

16 Zhang Q, Bastard P, Liu Z, et al. Inborn errors of type I IFN immunity in patients with life-threatening COVID-19. Science 2020;370:eabd4570.

17 Bastard P, Rosen LB, Zhang Q, et al. Auto-antibodies against type I IFNs in patients with life-threatening COVID-19. Science 2020;370:eabd4585.

18 van der Made Cl, Simons A, Schuurs-Hoeijmakers J, et al. Presence of genetic variants among young men with severe COVID-19. JAMA 2020. doi:10.1001/ jama.2020.13719. [Epub ahead of print: 24 Jul 2020].

19 Novelli A, Andreani M, Biancolella M, et al. Hla allele frequencies and susceptibility to COVID-19 in a group of 99 Italian patients. HLA 2020;96:610-4.

20 Benetti E, Tita R, Spiga O, et al. Ace2 gene variants may underlie interindividual variability and susceptibility to COVID-19 in the Italian population. Eur I Hum Genet 2020;28:1602-14 
21 Novelli A, Biancolella M, Borgiani P, et al. Analysis of ACE2 genetic variants in 131 Italian SARS-CoV-2-positive patients. Hum Genomics 2020;14:29.

22 Gómez J, Albaiceta GM, García-Clemente M, et al. Angiotensin-Converting enzymes (ACE, ACE2) gene variants and COVID-19 outcome. Gene 2020;762:145102.

23 Zhou R, To KK-W, Wong Y-C, et al. Acute SARS-CoV-2 infection impairs dendritic cell and T cell responses. Immunity 2020;53:864-77.

24 Odak I, Barros-Martins J, Bošnjak B, et al. Reappearance of effector T cells is associated with recovery from COVID-19. EBioMedicine 2020;57:102885.

25 Mazzoni A, Salvati L, Maggi L, et al. Impaired immune cell cytotoxicity in severe COVID-19 is IL-6 dependent. J Clin Invest 2020;130:4694-703.

26 Kuri-Cervantes L, Pampena MB, Meng W, et al. Comprehensive mapping of immune perturbations associated with severe COVID-19. Sci Immunol 2020;5:eabd 7114-abd7114.

27 Wen W, Su W, Tang H, et al. Immune cell profiling of COVID-19 patients in the recovery stage by single-cell sequencing. Cell Discov 2020;6:31.

28 Lucas C, Wong P, Klein J, et al. Longitudinal analyses reveal immunological misfiring in severe COVID-19. Nature 2020:584:463-9.

29 Westmeier J, Paniskaki K, Karakose Z. Impaired cytotoxic CD8+ T cell response in elderly COVID-19 patients. mBio2020:11.

30 De Biasi S, Meschiari M, Gibellini L, et al. Marked T cell activation, senescence, exhaustion and skewing towards Th17 in patients with COVID-19 pneumonia. Nat Commun 2020;11:3434.

31 Song $C-Y, X u J$, He J-Q, et al. Immune dysfunction following COVID-19, especially in severe patients. Sci Rep 2020;10:15838.

32 Wang F, Hou H, Yao Y, et al. Systemically comparing host immunity between survived and deceased COVID-19 patients. Cell Mol Immunol 2020;17:875-7.

33 Arunachalam PS, Wimmers F, Mok CKP, et al. Systems biological assessment of immunity to mild versus severe COVID-19 infection in humans. Science 2020;369:1210-20.

34 Lee JS, Park S, Jeong HW, et al. Immunophenotyping of COVID-19 and influenza highlights the role of type I interferons in development of severe COVID-19. SCi Immunol 2020;5:eabd1554.

35 Silvin A, Chapuis N, Dunsmore G, et al. Elevated calprotectin and abnormal myeloid cell subsets discriminate severe from mild COVID-19. Cell 2020;182:1401-18.

36 Schulte-Schrepping J, Reusch N, Paclik D, et al. Severe COVID-19 is marked by a dysregulated myeloid cell compartment. Cell 2020;182:1419-40.

37 Wang Y, Zhang L, Sang L, et al. Kinetics of viral load and antibody response in relation to COVID-19 severity. J Clin Invest 2020;130:5235-44.

38 Xiang F, Wang X, He X. Antibody detection and dynamic characteristics in patients with COVID-19. Clin Infect Dis Off Publ Infect Dis Soc Am 2020.

39 Zhao J, Yuan Q, Wang H, et al. Antibody responses to SARS-CoV-2 in patients with novel coronavirus disease 2019. Clin Infect Dis 2020;71:2027-34.

40 Xie J, Ding C, Li J, et al. Characteristics of patients with coronavirus disease (COVID-19) confirmed using an IgM-IgG antibody test. J Med Virol 2020;92:2004-10.

41 Zhou M, Zhong J, Bi L, et al. Serological characteristics of COVID-19 patients. Infect Dis 2020;52:749-50.

42 Fajgenbaum DC, June CH. Cytokine storm. N Engl J Med Overseas Ed 2020;383:2255-73.

43 Chi Y, Ge Y, Wu B, et al. Serum cytokine and chemokine profile in relation to the severity of coronavirus disease 2019 in China. J Infect Dis 2020;222:746-54.

44 Sims JT, Krishnan V, Chang C-Y, et al. Characterization of the cytokine storm reflects hyperinflammatory endothelial dysfunction in COVID-19. J Allergy Clin Immunol 2021:147:107-11.

45 Xu Z-S, Shu T, Kang L, et al. Temporal profiling of plasma cytokines, chemokines and growth factors from mild, severe and fatal COVID-19 patients. Signal Transduct Target Ther 2020;5:100

46 Mojtabavi H, Saghazadeh A, Rezaei N. Interleukin-6 and severe COVID-19: a systematic review and meta-analysis. Eur Cytokine Netw 2020:31:44-9.

47 Karki R, Sharma BR, Tuladhar S. Synergism of TNF- $\alpha$ and IFN- $\gamma$ triggers inflammatory cell death, tissue damage, and mortality in SARS-CoV-2 infection and cytokine shock syndromes. Cell 2020

48 Leisman DE, Ronner L, Pinotti R, et al. Cytokine elevation in severe and critical COVID-19: a rapid systematic review, meta-analysis, and comparison with other inflammatory syndromes. Lancet Respir Med 2020;8:1233-44.

49 Di Minno A, Ambrosino P, Calcaterra I, et al. COVID-19 and venous thromboembolism: a meta-analysis of literature studies. Semin Thromb Hemost 2020;46:763-71.

50 Manne BK, Denorme F, Middleton EA. Platelet gene expression and function in COVID-19 patients. Blood2020

51 Hottz ED, Azevedo-Quintanilha IG, Palhinha L, et al. Platelet activation and plateletmonocyte aggregate formation trigger tissue factor expression in patients with severe COVID-19. Blood 2020;136:1330-41

52 Denorme F, Manne BK, Portier I, et al. COVID-19 patients exhibit reduced procoagulant platelet responses. J Thromb Haemost 2020;18:3067-73.

53 Zaid Y, Puhm F, Allaeys I, et al. Platelets can associate with SARS-Cov-2 RNA and are hyperactivated in COVID-19. Circ Res 202010.1161/CIRCRESAHA.120.317703. [Epub ahead of print: 17 Sep 2020].
54 Middleton EA, He X-Y, Denorme F, et al. Neutrophil extracellular traps contribute to immunothrombosis in COVID-19 acute respiratory distress syndrome. Blood 2020;136:1169-79.

55 Busch MH, Timmermans SAMEG, Nagy M, et al. Neutrophils and contact activation of coagulation as potential drivers of COVID-19. Circulation 2020;142:1787-90

56 Leppkes M, Knopf J, Naschberger E, et al. Vascular occlusion by neutrophil extracellular traps in COVID-19. EBioMedicine 2020;58:102925.

57 Nizzoli ME, Merati G, Tenore A, et al. Circulating endothelial cells in COVID-19. Am J Hematol 2020;95:E187-8.

58 Guervilly C, Burtey S, Sabatier F, et al. Circulating endothelial cells as a marker of endothelial injury in severe COVID -19. J Infect Dis 2020;222:1789-93.

59 Khider L, Gendron N, Goudot G, et al. Curative anticoagulation prevents endothelial lesion in COVID-19 patients. J Thromb Haemost 2020;18:2391-9.

60 Cugno M, Meroni PL, Gualtierotti R, et al. Complement activation in patients with COVID-19: A novel therapeutic target. J Allergy Clin Immunol 2020;146:215-7.

61 Xiao L-S, Zhang W-F, Gong M-C, et al. Development and validation of the HNC-LL score for predicting the severity of coronavirus disease 2019. EBioMedicine 2020;57:102880

62 Zhang C, Qin L, Li K, et al. A novel scoring system for prediction of disease severity in COVID-19. Front Cell Infect Microbiol 2020;10:318.

63 Li Q, Zhang J, Ling Y, et al. A simple algorithm helps early identification of SARS-CoV-2 infection patients with severe progression tendency. Infection 2020;48:577-84

64 Liang W, Liang H, Ou L, et al. Development and validation of a clinical risk score to predict the occurrence of critical illness in hospitalized patients with COVID-19. JAMA Intern Med 2020;180:1081.

65 Jehi L, Ji X, Milinovich A, et al. Development and validation of a model for individualized prediction of hospitalization risk in 4,536 patients with COVID-19. PLoS One 2020;15:e0237419.

66 Gerotziafas GT, Sergentanis TN, Voiriot G, et al. Derivation and validation of a predictive score for disease worsening in patients with COVID-19. Thromb Haemost 2020;120:1680-90.

67 Bartoletti M, Giannella M, Scudeller L, et al. Development and validation of a prediction model for severe respiratory failure in hospitalized patients with SARSCoV-2 infection: a multicentre cohort study (PREDI-CO study). Clin Microbiol Infect 2020;26:1545-53.

68 Weng Z, Chen Q, Li S, et al. ANDC: an early warning score to predict mortality risk for patients with coronavirus disease 2019. J Trans/ Med 2020;18:328.

69 Wu S, Du Z, Shen S. Identification and validation of a novel clinical signature to predict the prognosis in confirmed COVID-19 patients. Clin Infect Dis Off Publ Infect Dis Soc Am 2020

70 Xu R, Hou K, Zhang K, et al. Performance of two risk-stratification models in hospitalized patients with coronavirus disease. Front Med 2020;7:518

71 Ji M, Yuan L, Shen W, et al. A predictive model for disease progression in nonseverely ill patients with coronavirus disease 2019. Eur Respir J 2020;56:2001234.

72 Wang J, Zhang $\mathrm{H}$, Qiao R, et al. Thrombo-inflammatory features predicting mortality in patients with COVID-19: the FAD-85 score. J Int Med Res 2020:48:300060520955037.

73 Dong Y-M, Sun J, Li Y-X. Development and validation of a nomogram for assessing survival in patients with COVID-19 pneumonia. Clinical Infectious Diseases:395.

74 Caricchio R, Gallucci M, Dass C, et al. Preliminary predictive criteria for COVID-19 cytokine storm. Ann Rheum Dis 2021:80:88-95.

75 Li B, Zhang S, Zhang R, et al. Epidemiological and clinical characteristics of COVID-19 in children: a systematic review and meta-analysis. Front Pediatr 2020;8.

76 Kaushik S, Aydin SI, Derespina KR, et al. Multisystem inflammatory syndrome in children associated with severe acute respiratory syndrome coronavirus 2 infection (MIS-C): a multi-institutional study from New York City.J Pediatr 2020:224:24-9.

77 Morris SB, Schwartz NG, Patel P. Case series of multisystem inflammatory syndrome in adults associated with SARS-CoV-2 infection — United Kingdom and United States, March-August 2020. MMWR Morb Mortal Wkly Rep 2020:69.

78 Weisberg SP, Connors TJ, Zhu Y, et al. Distinct antibody responses to SARS-CoV-2 in children and adults across the COVID-19 clinical spectrum. Nat Immunol 2021:22:25-31

79 Mitjo, Corbacho-Monné M, Ubals M. Hydroxychloroquine for early treatment of adults with mild coronavirus disease 2019: a randomized, controlled trial. Clinical Infectious Diseases:14.

80 Skipper CP, Pastick KA, Engen NW, et al. Hydroxychloroquine in nonhospitalized adults with early COVID-19: a randomized trial. Ann Intern Med 2020;173:623-31.

81 Hu K, Wang M, Zhao Y. A small-scale medication of leflunomide as a treatment of COVID-19 in an open-label Blank-Controlled clinical trial. Virol Sin2020.

82 The RECOVERY Collaborative Group. Dexamethasone in hospitalized patients with Covid-19 — preliminary report. N Engl J Med Overseas Ed 2020:NEJMoa2021436.

83 Devaux CA, Rolain J-M, Colson P, et al. New insights on the antiviral effects of chloroquine against coronavirus: what to expect for COVID-19? Int J Antimicrob Agents 2020;55:105938.

84 Abd-Elsalam S, Esmail ES, Khalaf $\mathrm{M}$, et al. Hydroxychloroquine in the treatment of COVID-19: a multicenter randomized controlled study. Am J Trop Med Hyg 2020;103:1635-9 
85 Lyngbakken MN, Berdal J-E, Eskesen A, et al. Norwegian coronavirus disease 2019 (NO COVID-19) pragmatic open label study to assess early use of hydroxychloroquine sulphate in moderately severe hospitalised patients with coronavirus disease 2019: a structured summary of a study protocol for a randomised controlled trial. Trials 2020;21:485.

86 RECOVERY Collaborative Group, Horby P, Mafham M, et al. Effect of hydroxychloroquine in hospitalized patients with Covid-19. N Engl J Med 2020:383:2030-40.

87 Cavalcanti AB, Zampieri FG, Rosa RG. Hydroxychloroquine with or without azithromycin in mild-to-moderate Covid-19. N Engl J Med 2020;383:e119:2041-52.

88 Huang HD, Jneid H, Aziz M, et al. Safety and effectiveness of hydroxychloroquine and azithromycin combination therapy for treatment of hospitalized patients with COVID-19: a Propensity-Matched study. Cardiol Ther 2020;9:523-34.

89 Tang W, Cao Z, Han M, et al. Hydroxychloroquine in patients with mainly mild to moderate coronavirus disease 2019: open label, randomised controlled trial. BMJ 2020;369:m1849.

90 Tomazini BM, Maia IS, Cavalcanti AB, et al. Effect of dexamethasone on days alive and Ventilator-Free in patients with moderate or severe acute respiratory distress syndrome and COVID-19: the Codex randomized clinical trial. JAMA 2020:324:1307.

91 Jeronimo CMP, Farias MEL, Val FFA. Methylprednisolone as adjunctive therapy for patients hospitalized with COVID-19 (Metcovid): a randomised, double-blind, phase Ilb, placebocontrolled trial. Clin Infect Dis 2020. [Epub ahead of print: 12 Aug 2020].

92 Edalatifard M, Akhtari M, Salehi M, et al. Intravenous methylprednisolone pulse as a treatment for hospitalised severe COVID-19 patients: results from a randomised controlled clinical trial. Eur Respir J 2020;56. doi:10.1183/13993003.02808-2020. [Epub ahead of print: 24 Dec 2020].

93 Angus DC, Berry S, Lewis RJ, et al. The REMAP-CAP (randomized embedded multifactorial adaptive platform for community-acquired pneumonia) study. rationale and design. Ann Am Thorac Soc 2020;17:879-91.

94 Dequin P-F, Heming N, Meziani F, et al. Effect of hydrocortisone on 21-day mortality or respiratory support among critically ill patients with COVID-19: a randomized clinical trial. JAMA 2020;324:1298.
95 Hermine 0 , Mariette $X$, Tharaux P-L, et al. Effect of tocilizumab vs usual care in adults hospitalized with COVID-19 and moderate or severe pneumonia: a randomized clinical trial. JAMA Intern Med 2021;181:32-40.

96 Stone JH, Frigault MJ, Serling-Boyd NJ, et al. Efficacy of tocilizumab in patients hospitalized with Covid-19. N Engl J Med 2020;383:2333-44.

97 Salvarani C, Dolci G, Massari M, et al. Effect of tocilizumab vs standard care on clinical worsening in patients hospitalized with COVID-19 pneumonia: a randomized clinical trial. JAMA Intern Med 2021;181:24.

98 The REMAP-CAP Investigators. Interleukin-6 receptor antagonists in critically ill patients with Covid-19 - preliminary report. medRxiv 2020.

99 Gupta S, Wang W, Hayek SS, et al. Association between early treatment with tocilizumab and mortality among critically ill patients with COVID-19. JAMA Intern Med 2021:181:41-51.

100 Mariette X, Hermine O, Tharaux P-L. Effect of Anakinra vs usual care in adults hospitalized with COVID-19 and mild-to-moderate pneumonia: a randomized clinical trial. Lancet Respir Med2021.

101 Cavalli G, De Luca G, Campochiaro C, et al. Interleukin-1 blockade with highdose anakinra in patients with COVID-19, acute respiratory distress syndrome, and hyperinflammation: a retrospective cohort study. Lancet Rheumatol 2020;2:e325-31.

102 Huet T, Beaussier H, Voisin O, et al. Anakinra for severe forms of COVID-19: a cohort study. Lancet Rheumatol 2020;2:e393-400.

103 Iglesias-Julián E, López-Veloso M, de-la-Torre-Ferrera N, et al. High dose subcutaneous Anakinra to treat acute respiratory distress syndrome secondary to cytokine storm syndrome among severely ill COVID-19 patients. J Autoimmun 2020;115:102537.

104 Kalil AC, Patterson TF, Mehta AK, et al. Baricitinib plus Remdesivir for hospitalized adults with Covid-19. N Engl J Med 2020. doi:10.1056/NEJMoa2031994. [Epub ahead of print: 11 Dec 2020].

105 Landewé RB, Machado PM, Kroon F, et al. EULAR provisional recommendations for the management of rheumatic and musculoskeletal diseases in the context of SARS CoV-2. Ann Rheum Dis 2020;79:851-8. 